\title{
Apoio Logístico Integrado: Peculiaridades da Indústria de Defesa e Tecnologia
}

\author{
Integrated Logistics Support: \\ Peculiarities of the Defense and Technology Industry
}

Rev. Bra. Est. Def. v. 2, no 1, jan./jun. 2015, p. 53-72

ISSN 2358-3932

HEITOR FREIRE DE ABREU*

\section{INTRODUÇÃO}

$\mathrm{O}$ atual ressurgimento da chamada indústria de defesa ${ }^{1}$ no Brasil - que na década de 1980 praticamente desapareceu - teve como consequência significativa a criação e o fomento de empresas especializadas nessa área. Por se tratar de um segmento que integra tecnologia de ponta, conhecimento científico e pesquisa avançada, há necessidade de aporte financeiro expressivo, capacidade de gerenciar projetos de alto risco, recursos humanos extremamente especializados, experiência em negócios internacionais e capacidade empreendedora sólida e diversificada para se atuar nesse campo.

Fruto desse cenário, surgiram no mundo e no Brasil as chamadas empresas integradoras que desempenham o papel de main contractors ${ }^{2}$ em projetos complexos, como o Prosub ${ }^{3}$. Dentre as diversas engenharias ${ }^{4}$ envolvidas nesse ciclo, destacam-se as de sistemas, eletrônica, mecânica, integração de software (SW) e hardware (HW), segurança, produção, confiabilidade, testagem, qualidade e logística integrada.

Este artigo tem como objetivo abordar o papel dessa última na indústria de defesa e tecnologia, o chamado Apoio Logístico Integrado (ALI) ou, como é mais conhecido, pela expressão em inglês, Integrated Logistics Support (ILS).

\footnotetext{
* Tenente-coronel da reserva do Exército Brasileiro e doutor em Ciências Militares (ECEME). Analista da Odebrecht Defesa e Tecnologia (ODT). E-mail: majheitor@gmail.com.
} 
RBED, v. 2, no 1 , jan./jun. 2015

Figura 1 - Exemplos de engenharias ou áreas de uma empresa integradora de defesa e tecnologia

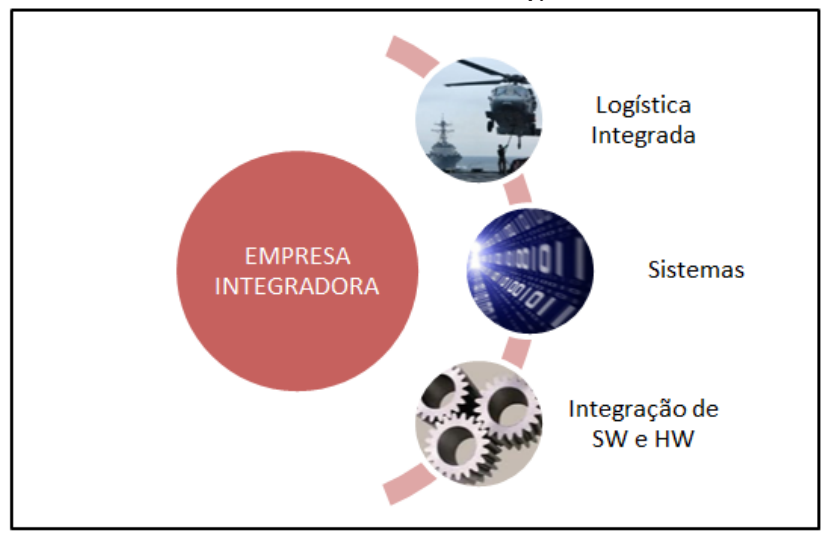

Fonte: elaboração própria.

\section{A INDÚSTRIA DE DEFESA E TECNOLOGIA}

Segundo Correa Filho et al (2013), a indústria de defesa tem sua estrutura oligopolizada, com os principais atores oriundos de grandes conglomerados e com atuação diversificada também fora desses mercados. Como exemplo, têm-se, entre as 10 maiores empresas de defesa do mundo, sem incluir as de origem chinesa, os conglomerados Lockheed Martin, Boeing, BAE Systems, Raytheon, General Dynamics, Northrop Grumman, EADS, United Technologies, UTC, Finmeccanica e L-3 Communications. Todas as empresas listadas possuem negócios que vão além da área de defesa e tecnologia. Para que se tenha uma ideia do potencial econômico dessa indústria, somente as 10 citadas anteriormente empregaram, segundo dados de 2012 do Stockholm International Peace Research Institute 5 (SIPRI), mais de um milhão e oitenta e sete mil pessoas, tiveram um faturamento de USD 408,2 bilhões e um lucro de USD 20,7 bilhões, em valores aproximados. (Fleurant; Perlo-Freeman, 2014). 
Tabela 1 - Comércio exterior mundial de equipamentos militares por sistemas (2003-2012)

\begin{tabular}{|l|c|}
\hline \multicolumn{1}{|c|}{ Sistema } & Valor em USD milhões de 1990 \\
\hline Aeronaves & 108.088 \\
\hline Navios & 37.662 \\
\hline Mísseis & 32.694 \\
\hline Veículos Blindados & 29.310 \\
\hline Sensores & 13.049 \\
\hline Sistemas de Defesa Antiaérea & 10.284 \\
\hline Motores & 9.822 \\
\hline Artilharia & 4.822 \\
\hline Satélite & 50 \\
\hline Armas antissubmarino & 28 \\
\hline Outros & 1.857 \\
\hline Total & 247.090 \\
\hline
\end{tabular}

Fonte: elaboração própria, com dados extraídos de Correa Filho et al (2013).

O mercado de defesa e tecnologia no Brasil é caracterizado por ter um orçamento incerto, dependente de aportes orçamentários e - principalmente - extra orçamentários, necessitar de financiamento interno e externo para as empresas do segmento, além de pensar em produtos com perspectivas de exportação. Objetivamente, defesa e tecnologia pressupõem aquisições estratégicas, de longo prazo, com políticas públicas específicas, calcadas na área de pesquisa com grande aporte no meio acadêmico, visando a aquisição e a transferência de tecnologia. Para isso, o fomento à chamada Base Industrial de Defesa (BID) ${ }^{6}$ está fundamentado em fatores orçamentários, de financiamento (indústria e pesquisa \& desenvolvimento) e exportação e mecanismos de transferência de tecnologia ${ }^{7}$ (entenda-se compensação comercial e cooperação industrial).

No Brasil, a interlocução entre as empresas de defesa e tecnologia e o governo é realizada, via de regra, por intermédio do Ministério da Defesa (MD), mais precisamente, por meio da Secretaria de Produtos de Defesa (SEPROD) e do seu Departamento de Produtos da Defesa (DEPROD). Esse canal, recentemente criado, facilita, sobremaneira, a troca de informações, o esclarecimento de dúvidas e a formulação de políticas voltadas para esse mercado. Essa abordagem é importante na medida em que a estratégia de defesa é inseparável da estratégia de desenvolvimento do país, visando, em última análise, os projetos de independência tecnológica do Brasil, a mobilização de recursos e a capacitação. 
RBED, v. 2, no 1 , jan./jun. 2015

\section{SISTEMAS DE DEFESA DE ALTA COMPLEXIDADE TECNOLÓGICA}

Antes de se abordar o ALI para os sistemas de defesa de alta complexidade tecnológica, é importante que se entenda melhor o seu significado e como tais sistemas são desenvolvidos. As melhores práticas da engenharia de sistemas sugerem que se utilizem modelos para o seu incremento. Dentre os existentes, destacam-se o Waterfall (um dos primeiros a serem utilizados), o Spiral e o Vee, dentre outros. Para que se tenha uma ideia das áreas onde esses modelos atuam e como são suas divisões, apresenta-se a abordagem conhecida como top-down/botton-up, onde se pode enxergar o que é um desenvolvimento de um sistema complexo e delineia-se a fase de design e construção (top-down) e a fase de integração e utilização (botton$-u p)$.

Figura 2 - Estrutura top-down/botton-up

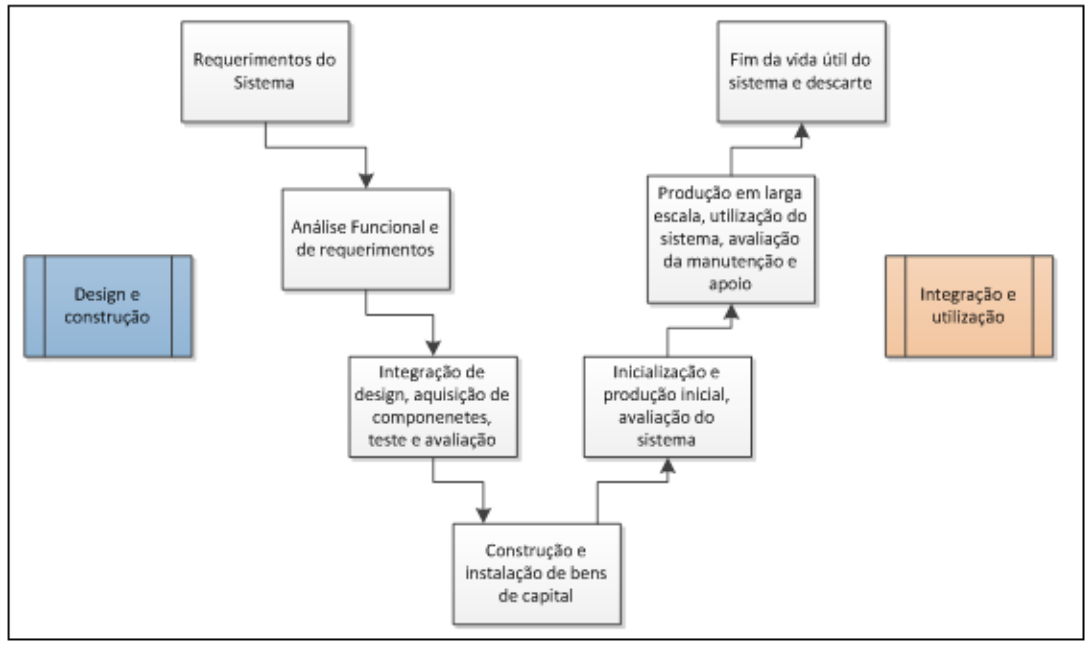

Fonte: (Blanchard, 2008).

Pode-se ver na figura 3 as interações e a necessidade de um apoio logístico robusto para manter um sistema complexo de defesa operando por anos. 
Figura 3 - Interação Sistema x ALI

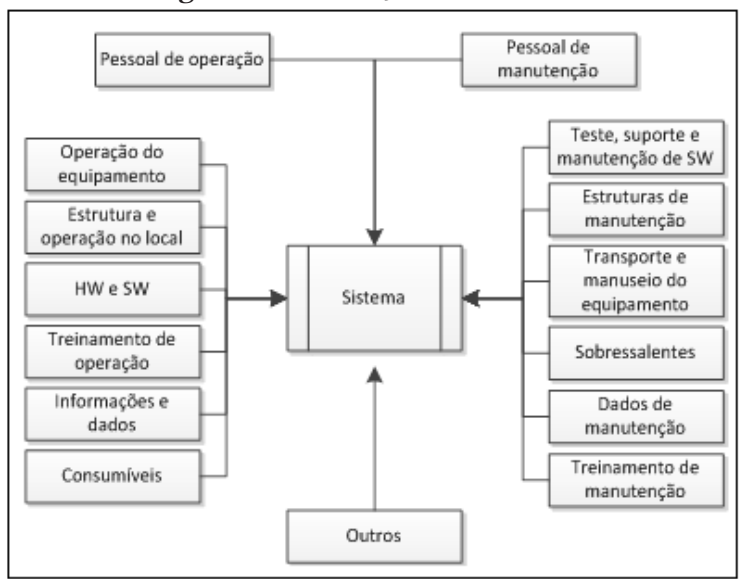

Fonte: (Blanchard, 2008).

A figura 4 ilustra como sistemas de defesa demandam grande número de recursos humanos especializados, enorme alocação de horas de trabalho e uma considerável quantidade de peças a serem fabricadas e catalogadas. Utilizando-se o exemplo do submarino a propulsão nuclear brasileiro (SN-BR), em fase de desenvolvimento e fabricação, vê-se que serão consumidas 12 milhões de horas/homem $(\mathrm{HH})$ de trabalho e fabricadas cerca de 1.000.000 (um milhão) de peças. Quando pronto, em 2025, este sistema - o SN-BR - demandará enorme apoio logístico, integrando manutenção, treinamento, sobressalentes, catalogação e outras áreas do ALI a fim de mantê-lo em funcionamento, malgrado as duras condições de operação ${ }^{8}$.

Figura 4 - Complexidade para Construção de Sistemas de Defesa e Tecnologia

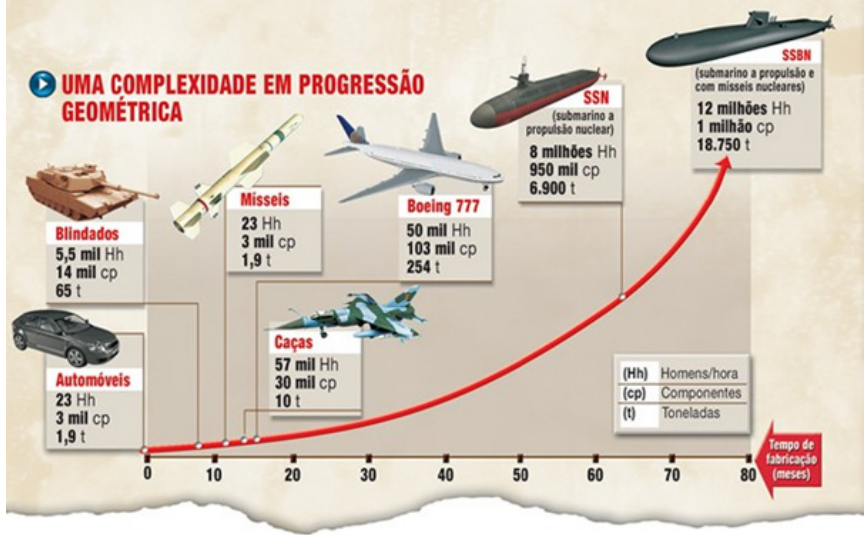

Fonte: Diário do Pré-Sal (2010). 
RBED, v. 2, no 1, jan./jun. 2015

\section{O APOIO LOGÍSTICO INTEGRADO PARA SISTEMAS DE DEFESA DE ALTA COMPLEXIDADE TECNOLÓGICA}

O ALI ou ILS, notadamente na área militar, surgiu em função das características peculiares dos grandes projetos: alto valor agregado dos sistemas, subsistemas e componentes, grande complexidade tecnológica e necessidade de mantê-los funcionando em um horizonte temporal de médio e longo prazos.

A definição utilizada nesse artigo para ALI, original nossa, é: o gerenciamento do ciclo de vida, por meio de metodologias peculiares, que atenda às fases de concepção, viabilidade, definição, desenvolvimento, fabricação, implantação, operação, revitalização e desativação, assegurando que um sistema ou equipamento atenda aos requisitos funcionais e não-funcionais do projeto, a um preço aceitável pelos interessados e que possa ser apoiado logisticamente durante todo o seu ciclo de vida.

Além disso, destaca-se que o ALI envolve conhecimentos muito específicos dos possíveis clientes no Brasil: Marinha do Brasil, Exército Brasileiro, Força Aérea Brasileira e Ministério da Defesa, por intermédio da Secretaria de Logística, Mobilização, Ciência e Tecnologia (SELOM). O conhecimento acerca de normas como SISMICAT, MIL-STD e EURO, dentre outras, é, também, fundamento e diferencial importante para dar o suporte técnico necessário aos equipamentos de defesa a serem adquiridos e integrados pela main contractor.

Atualmente, o Departamento de Defesa dos Estados Unidos da América é o grande referencial na área de logística integrada voltada para a área militar. Sua experiência e expertise adquiridas nos conflitos contemporâneos introduziram uma metodologia para o planejamento de uma logística integrada enxuta e eficiente, por meio de suas normas de padronização ${ }^{10}$, como Defense Handbook (MIL-HDBK), Performance Specification (MIL-PRF), Defense Standard (MIL-STD), Defense Specification (MIL-SPEC) e Detail Specification (MIL-DTL).

Os projetos militares durante a Guerra Fria utilizaram, ainda que de maneira empírica, esse conceito. No entanto, foram os projetos pós-Guerra Fria que obrigaram os militares e os civis a pensar em uma metodologia própria, haja vista a necessidade de se fazer o trade off entre a natural redução dos gastos em defesa e sistemas de armas cada vez mais complexos tecnologicamente e, consequentemente, mais caros. É o caso do avião de caça norte-americano F-22 Raptor, cujo preço unitário é estimado em US\$ 
361 milhões e demorou, desde o seu primeiro voo até entrar em combate atacando alvos do Estado islâmico da Síria, 17 anos (Godoy, 2014).

Nesse ponto, a Logística Integrada se firmou na área militar como uma solução para viabilizar logisticamente os projetos. A assinatura da Declaração de Intenções entre o Ministério da Defesa da República Federativa do Brasil e o Serviço Federal de Cooperação Técnico-Militar da Federação Russa Relativa à Cooperação em Defesa Antiaérea confirma essa assertiva, onde se destaca que

[d]eclaram a intenção de: a) [...]; e b) Incrementar, a partir de março de 2013, as negociações bilaterais com vistas à possibilidade de preparação de contrato para futuras obtenções, por parte do Governo do Brasil, de Baterias Antiaéreas, com o desenvolvimento conjunto de novos Produtos de Defesa e a participação de Empresas Estratégicas de Defesa brasileiras nos processos produtivos e de sustentabilidade logística integrada ${ }^{11}$, com transferência efetiva de tecnologia, sem restrições. (Brasil, 2013).

Dessa forma, a logística integrada é aspecto importante quando se trata de indústria de defesa, já que está presente em todo o processo de aquisição de um sistema, inclusive no seu design. Destaca-se que o ALI é transversal a todas as fases de uma compra de um sistema desse tipo, variando o seu impacto em cada uma delas e deve seguir as normas vigentes para cada cliente. Desde o momento em que uma força armada emite um request for information $(\mathrm{RFI})^{12}$, passando por um request for proposal $(\mathrm{RFP})^{13}$ até o descomissionamento, que poderá ocorrer entre 10 e 35 anos após o início da operação do sistema, como é o caso de um submarino ou um carro de combate.

\section{PECULIARIDADES DO ALI NA ÁREA DE DEFESA E TECNOLOGIA}

A logística integrada na área de defesa e tecnologia possui peculiaridades em relação à logística tradicional. Isso se deve ao fato que ela deve atender aos manuais, normas e aos requisitos militares de cada país. Além das particularidades de projetos que envolvem requisitos de segurança expressivos, há confiabilidade e service level agreement ${ }^{14}$ (SLA) altos, o que implica em uma divisão em subáreas ou disciplinas específicas.

Um exemplo dessas necessidades de customização é a Força Aérea Brasileira $(\mathrm{FAB})$ que, via de regra, utiliza como anexo às suas RFP um documento denominado Requisitos Técnicos, Logísticos e Industriais (RTLI), que não é usado por nenhum outro cliente. Serão, portanto, abordadas al- 
gumas áreas, dentre as muitas existentes, a fim de abordar as peculiaridades do ALI na área de defesa e tecnologia.

\subsection{Manutenção}

A manutenção após o início da operação é fundamental para que um sistema de defesa se mantenha em funcionamento durante o período esperado e dentro das condições contratuais acordadas. A maioria das Forças Armadas dividem a manutenção em quatro níveis ou escalões, em divisão apresentada no Manual de Logística da Marina do Brasil (Brasil, 2003):

- $1^{\circ}$ escalão: ações realizadas pelo usuário e/ou pela organização militar (OM) detentora do material ou software, com os meios orgânicos disponíveis, visando a mantê-los em condições de funcionamento e de conservação.

- $2^{\circ}$ escalão: ações realizadas em organizações de manutenção e que ultrapassam a capacidade dos meios orgânicos da OM responsável pelo material ou software. No caso dos sistemas digitais, são manutenções onde não há necessidade de alteração de código-fonte do software utilizado.

- $3^{\text {o }}$ escalão: ações de manutenção que exigem recursos superiores aos escalões anteriores, em função do grau de complexidade.

- $4^{\circ}$ escalão: ações de manutenção cujos recursos necessários, normalmente, ultrapassam a capacidade da força armada, sendo, na maioria das situações, executadas pelo fabricante ou representante autorizado, ou, ainda, necessitam do uso de instalações especializadas.

Já as empresas de defesa e tecnologia dividem seus níveis de manutenção de maneira um pouco diferente. Normalmente, nos EUA e Europa, utiliza-se 3 níveis: "O" level (organizational); "I" level (intermediate); e "D" level (depot). Em alguns casos, o $4^{\circ}$ escalão das Forças Armadas brasileiras e de outras é conhecido como "F" level (fabric), ou contractor level. Existe, ainda, a classificação da Association Française de Normalisation ${ }^{15}$ (AFNOR), que divide a manutenção em 5 níveis, segundo a norma FD X50-605, levando em consideração o serviço executado e o tipo de capacitação de quem executa a manutenção.

As atividades ou tipo de manutenção que a defesa normalmente utiliza são:

- manutenção preventiva e preditiva;

- manutenção modificadora; e

- manutenção corretiva ou reparo (planejada ou não-planejada).

Resumidamente, os níveis e as atividades de manutenção buscam manter o sistema no nível mais baixo possível (sempre que possível, $1^{\circ}$ e $2^{\circ}$ escalão, 
ou "O" e "I" levels), objetivando que seja realizado no sítio do equipamento ou o mais perto possível, realizando o equilíbrio entre a diminuição do tempo de reparo do equipamento e a disponibilidade de equipamentos (bens de capital) para a realização da manutenção. Em síntese, busca-se a maior redução de custo possível na disciplina manutenção. Adaptar nomenclaturas de normas diferentes às necessidades do cliente e às estruturas existentes dos fornecedores e da main contractor é a chave para se obter o menor custo.

\subsection{Transporte}

O transporte, como uma das áreas do ALI, reveste-se de peculiaridades importantes. Destaca-se a necessidade de se pensar em multimodalidade, pois muitos sistemas têm sítios localizados em áreas de difícil acesso, como ilhas, montanhas e outros lugares onde o acesso é ruim ou, muitas vezes, sequer existe. $\mathrm{O}$ uso de helicópteros, aviões ou caminhões especializados pode ser uma necessidade, o que encarece o transporte em alguns casos. Além disso, no Brasil, como as distâncias são expressivas, o uso de centros de distribuição descentralizados pode implicar na necessidade de se estabelecer subcontratos com operadores logísticos locais, principalmente na last mile ${ }^{16}$.

\subsection{Treinamento}

Realizar um treinamento de qualidade é uma necessidade quando se trabalha com equipamentos de defesa e tecnologia cujo valor agregado é enorme e o SLA é alto. Normalmente, os treinamentos são divididos em dois grandes segmentos: treinamento inicial e treinamento de manutenção.

O primeiro caracteriza-se por treinar pessoal para operação e manutenção do equipamento que será adotado por uma Força Armada. Ele é realizado com pessoal militar e civil e visa formar uma massa inicial de recursos humanos certificados para as ações de operação e manutenção assim que o sistema entra em operação.

Já o segundo é realizado tantas vezes quantas forem necessárias e tem como objetivo propiciar à Força Armada pessoal qualificado durante todo o ciclo de vida do sistema, mantendo uma massa crítica em condições de substituir as naturais evasões. É contínuo e tem seu início somente após o término do treinamento inicial. Busca repor recursos humanos perdidos em função de transferências, promoções, inatividade (aposentadoria) e outras fugas de capital humano. 
O treinamento pode - e deve - ser subdividido em categorias específicas em função do que se espera que o aluno certificado realize após o término do treinamento, bem como do nível de conhecimento anterior e formação necessária para se adquirir o conhecimento esperado. As categorias mais comuns são: operador, mantenedor, supervisor e instrutor.

\section{Tabela 2 - Fases do Treinamento}

\begin{tabular}{|c|c|c|c|c|}
\hline Conceito & Desenvolvimento & $\begin{array}{c}\text { Design } \\
\text { detalhado }\end{array}$ & Produção & Entrega \\
\hline $\begin{array}{c}\text { Planejamento do Treinamento } \\
\text { Inicial }\end{array}$ & Preparação & Treinamento & Treinamento \\
\hline \multicolumn{2}{|c|}{ Planejamento do Treinamento de Manutenção } & Preparação & Treinamento \\
\hline
\end{tabular}

\subsection{Latalogaçao}

Fonte: (Jones, 2006)

Se na área civil catalogar é um aspecto significativo, essa preocupação aumenta sobremaneira na área militar. Com a finalidade de mitigar os problemas advindos da enorme quantidade de peças e partes que formam um sistema complexo de defesa, a maioria dos países adota o sistema da Organização do Tratado do Atlântico Norte (OTAN). Isso se deve ao fato de que catalogar reduz custos do projeto, principalmente durante a fase de aquisição e operação do sistema, e facilita o apoio logístico.

Basicamente, o Sistema OTAN de Catalogação (SOC) tem por objetivo aumentar a eficiência dos sistemas logísticos, facilitar o manuseio de dados, minimizar os custos logísticos das organizações usuárias e aumentar a eficiência nas operações logísticas. Assim, quando um item de suprimento é estabelecido como único, em função do seu design, características físico-químicas ou outras, ele recebe o chamado Nato Stock Number (NSN), composto de 13 dígitos numéricos.

No Brasil, o órgão responsável pela catalogação perante à OTAN é o Centro de Catalogação das Forças Armadas (CECAFA), a quem cabe atribuir NSN aos itens de suprimento. Destaca-se que para cada item a ser catalogado há um valor cobrado.

\subsection{Sobressalentes ou Spare Parts}

O sobressalente, ou spare part, é aspecto importante no dimensionamento do ALI, pois implica em se analisar áreas como custo dos lotes anuais de sobressalentes, o que facilita a análise do trade off entre $\mathrm{CAPEX}^{17} \mathrm{e}$ $\mathrm{OPEX}^{18}$. Leva-se em conta, nessa análise, o horizonte temporal do ALI, a gestão de obsolescência de cada sistema, a necessidade de cada área de ar- 
mazenamento (centro de distribuição - CD), a disposição geográfica desses centros de distribuição e o lead time, haja vista que muitos desses sobressalentes não são $\mathrm{COTS}^{19}$, sendo, portanto, adquiridos por meio de encomendas planejadas com antecedência. Não se deve deixar de lado, também, as manutenções necessárias (preventiva, preditiva, corretiva e outras) em sintonia com o SLA acordado, a fim de se evitar multas contratuais.

A figura 5 mostra, de maneira genérica, o ciclo de uma manutenção corretiva como exemplo do impacto das spares parts.

Figura 5 - Fluxograma de Manutenção

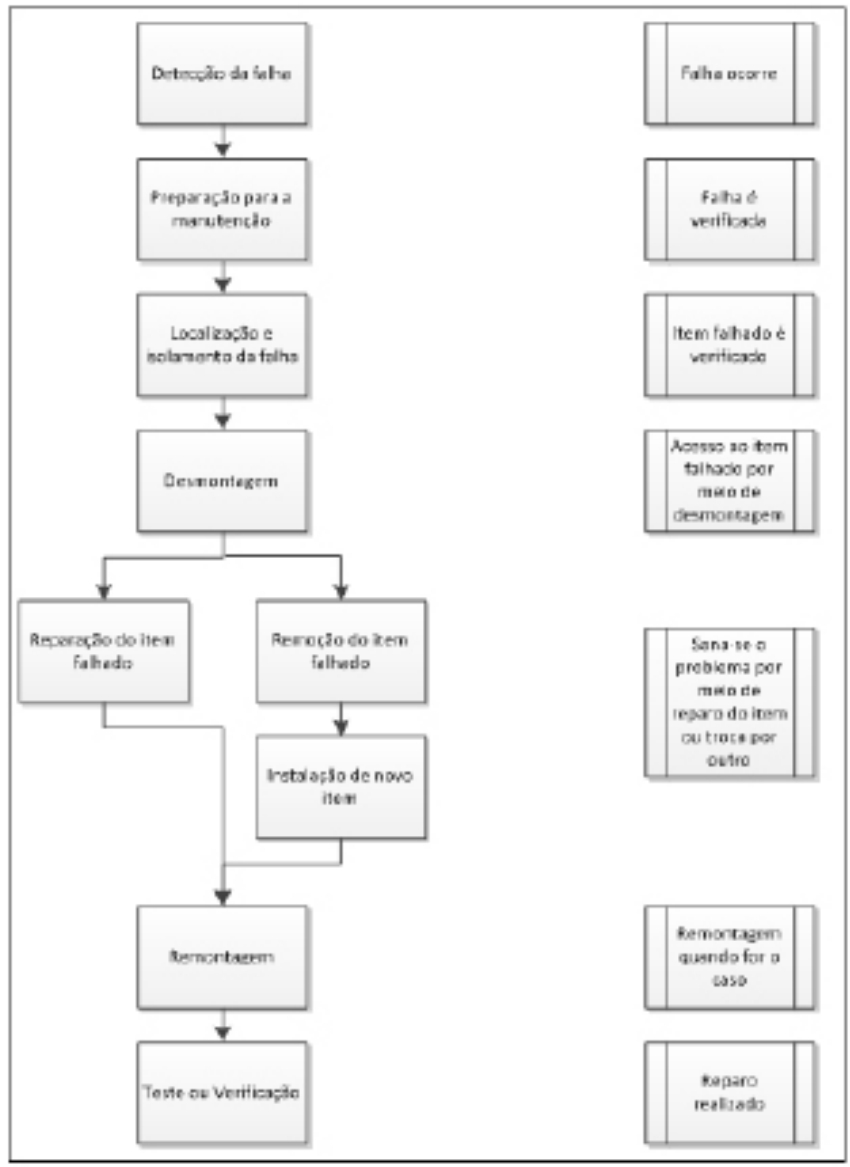

Fonte: (Jones, 2006). 
Para seguir esse fluxograma e fazer uma previsão econômica que tenha impacto na OPEX, em termos logísticos, de sobressalentes é preciso levar em consideração muitos parâmetros matemáticos e estatísticos, desde o desenvolvimento do produto até seu descomissionamento. Nesse sentido, conhecer a confiabilidade do sistema a ser adquirido é fundamental para a escolha daquele que melhor vai atender às necessidades de custos e de qualidade. A figura 6 mostra o conceito de confiabilidade.

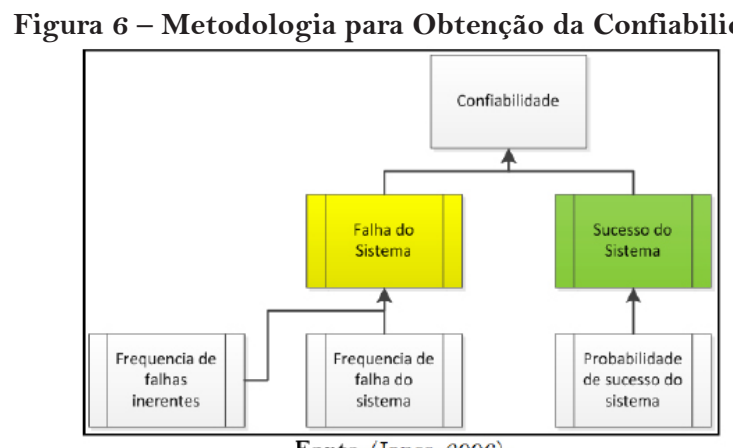

Fonte: (Jones, 2006)

Os principais parâmetros estatísticos são o mean time to repair (MTTR), que está ligado aos requisitos de desenvolvimento ou aquisição de um sistema; o mean corrective maintenance time (MCT), que considera aspectos como tempo (agenda e janela temporal) de acesso ao sistema e preparação para a manutenção (no gerenciamento do ALI de submarinos, por exemplo, utiliza-se o MCT em muitas manutenções no momento em que ele é docado) e outros aspectos que não podem ser considerados no MTTR; e o mean preventive maintenace time (MPMT), muito ligado ao $\mathrm{RCM}^{21}$, que busca antever uma possível falha prevista e que deve ser feita em função de um calendário previamente alinhado com os interessados.

Figura 7 - Cálculo da Taxa de Falha

$$
\lambda_{1}=\frac{\text { Número de falhas }}{\text { Total de horas de uso }}
$$

Fonte: elaboração própria.

Figura 8 - Cálculo da Taxa de Falha

MTTR $=\sum\left(\lambda_{1} \times\right.$ tempo requerido para reparo ou troca $) / \sum \lambda_{1}$

Fonte: elaboração própria. 
Além desses parâmetros utilizados na estimativa de confiabilidade de um sistema, existe o mean time between failure (MTBF), cuja finalidade é propiciar a taxa de falhas entre uma falha e outra.

Figura 9 - Cálculo do MTBF

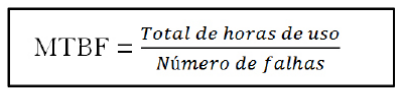

Fonte: elaboração própria.

Após a aplicação dessas e de muitas outras fórmulas e análises, o profissional da área de ALI tem condições de realizar a chamada failure modes, effects and criticality analysis (FMECA ${ }^{22}$ ), cujo objetivo principal é a completa análise de cada nível do sistema que fornecerá dados não só para o ALI, mas também para diversas áreas de engenharia. A FMECA analisa aspectos tais como: modo potencial de falha; efeitos potenciais de falha; severidade do efeito; causas potenciais da falha; ocorrência; controles atuais; detecção de falhas; número de prioridade de risco (NPR); ações recomendadas; e número de prioridade de risco revisado. Basicamente, verificará se o ALI está respondendo aos requisitos de alta qualidade e baixo custo que se espera em projetos de defesa e tecnologia.

Mudanças podem ser feitas nos parâmetros da FMECA a fim de atender o trade off qualidade x custo, equilibrando-se o sistema de acordo com os requisitos de performance, principalmente durante a operação, e o orçamento disponível para a aquisição do sistema.

\section{ASPECTOS A SEREM OBSERVADOS}

Nesta seção, elenca-se aspectos que merecem atenção do gerente de ALI, a fim de mostrar que a logística na área de defesa e tecnologia possui nuanças; isto é: (a) não devem ser replicadas por completo da logística tradicional; e (b) um bom gerente deve conhecer todo o projeto, possuir conhecimentos que extrapolam a logística de per se e ser capaz de concatenar aspectos que aparentemente não são da sua área a fim de evitar erros no projeto e aumento de custos na área de ALI

\subsection{Service Level Agreement (SLA)}

Os SLA da indústria de defesa e tecnologia são altos, já que defesa infere em monitoramento e capacidade de atuar $24 \times 7$, durante os 365 dias do ano. 
Assim, para atender a esse parâmetro básico de prontidão, a conjugação de SLA acima de $95 \%$ para todo o sistema com redundâncias lógicas e físicas torna-se primordial.

Essa característica no SLA da defesa e tecnologia pode encarecer o produto final, o que implica na existência de um help desk robusto, com um service desk extremamente flexível, grande capilaridade geográfica dos fornecedores e da main contractor, além de intenso uso de comunicações rápidas (redes de internet, telefonia móvel e outros).

\subsection{Centros de Distribuição (CD)}

Os CD na área de defesa e tecnologia possuem as características usuais dos CD comerciais acrescidas de medidas de segurança e de ambiente ${ }^{23} \mathrm{e}$ de procedimentos para acesso ao material armazenado muito restritos. Isso se deve ao fato que eles estocam spare parts de alto valor agregado e que, muitas vezes, são controlados por legislações internacionais rígidas, a fim de não serem de uso ou posse de pessoas ou grupos não autorizados ou, até mesmo, perigosos.

Caracterizam-se, também, por consumir pouca área em função de os equipamentos sobressalentes serem de pequena dimensão e de alto valor agregado, possuir MTBF alto, diminuindo a necessidade de se armazenar grandes lotes de spare parts e apoiarem poucas bases, já que, via de regra, é preferível se possuir vários CD próximos aos sistemas (vide níveis de manutenção) do que centralizar em locais distantes, o que dificultaria a manutenção do SLA contratado.

\subsection{Alta Rotatividade de Operadores e Manutenção}

No que tange à manutenção e à operação, um problema a ser equacionado pelo gerente de ALI em projetos de defesa e tecnologia é a grande rotatividade de operadores e mecânicos nos diversos níveis. Essa rotatividade se dá em função de promoções do pessoal militar, transferências para outras cidades, mudança de função dentro da mesma unidade, passagem para a reserva ${ }^{24}$ e outras injunções específicas da profissão militar.

Para mitigar esse problema, sugere-se: treinar, sempre que possível, pessoal militar recém formado, por serem menos suscetíveis às transferências; planejar treinamentos em curtos espaços de tempo; utilizar replicadores de conhecimento; formar um percentual um pouco maior do que o necessário para servir de stand by; prever cursos nas escolas de formação e aperfeiçoamento militares a fim de propiciar uma massa crítica com co- 
nhecimento básico ${ }^{25}$; e procurar sugerir às Forças Armadas políticas de retenção de pessoal nos locais em que os sistemas estão baseados. É sempre importante lembrar que rotatividade significa, em uma primeira análise, em aumento de custos com cursos, certificações, incremento na quebra de equipamentos por falta de experiência dos operadores, desgaste prematuro de componentes e deslocamentos de pessoal ${ }^{26}$.

\subsection{Incentivo à Formação de Especialistas em Instituições de Ensino de Nível Superior}

Durante a realização do ALI, desde a fase de concepção até a desativação, muitos especialistas em diversas áreas serão necessários. Como o gerente do ALI é responsável pelos treinamentos e preparação dos recursos humanos para manter o projeto em andamento, é fundamental que ele compreenda o papel das instituições de nível superior, notadamente da pós-graduação, com a finalidade de se transferir conhecimento e tecnologia para o país. Estimular, seja na forma de custos em treinamento do próprio contrato, seja como opção de off set, a ida de pessoal para universidades e instituições de ensino no exterior a fim de conhecer e dominar áreas como acústica submarina, integração de sistemas, programação em linguagens específicas, soldagens especiais, optrônica, telecomunicações, radares e balística, dentre muitas outras, é fundamental para agregar valor ao projeto e, principalmente, para trazer ao país conhecimento de alto nível para o meio acadêmico.

A participação de Institutos de Ciência e Tecnologia (ICT) complementa esse quadro na medida que passam a ser depositários dos conhecimentos adquiridos, conduzindo-os para novas fronteiras do conhecimento, cumprindo o papel que a defesa sempre teve, historicamente, em maior ou menor grau, no desenvolvimento de tecnologias de ponta e no estado da arte a fim de serem utilizadas em áreas civis que propiciem bem-estar para a população.

\section{CONCLUSÃO}

Desenvolver e integrar sistemas, subsistemas e componentes que envolvam tecnologias como guiamento a laser, radares, propulsão naval e aérea, software, hardware, acústica submarina, balística, química e mecânica fina, sensores eletrônicos e combustíveis propelentes, dentre outros, não é tarefa simplória. Esse desafio não pode ser delegado a empresas cuja 
robustez, experiência e capacidade em lidar com as naturais flutuações desse mercado tão exigente, e com crescimento exponencial de tecnologias e velocidade de resposta, não tenha sido sobejamente comprovada.

Para suportar e apoiar esse desafio tecnológico é preciso uma logística especializada, experiente e com capacidade de compreender que, embora o fulcro desses projetos seja o sistema em si, o ALI é quem o manterá funcionando, notadamente após a implantação.

Quando se estuda o negócio de defesa e tecnologia e os naturais clientes do segmento, conclui-se que a logística integrada, um pouco diferente do que ocorre na área industrial, não se encerra com a entrega do projeto. A relação entre a integradora e o cliente continua por algo em torno de 5 a 35 anos, tendo a logística integrada como grande maestra. O ALI se faz presente por meio de suas expertises, tais como manutenção, treinamento, assistência técnica, garantia e transporte, dentre outras.

Por fim, é fundamental que se compreenda o papel da indústria de defesa e tecnologia na história do desenvolvimento das ciências no Brasil - seja na área conhecida como dura ou tradicional (Matemática, Física e Química, dentre outras); seja em áreas distintas da primeira (Computação, Biologia e Economia, dentre outras) - como indutor de campos do conhecimento que desaguam em avanços que colaboram para a melhoria do bem-estar social e incremento de outras ciências em vertentes extremamente multidisciplinares. A logística integrada na área de defesa e tecnologia, inserida nesse papel, quando aperfeiçoa ferramentas, inova abordagens, diminui custos por meio de soluções de gestão e desenvolvimento de novas métricas estatísticas e parâmetros matemáticos, também auxilia o país a acrescentar novos conhecimentos que, quando mesclados com outros segmentos das ciências, agrega valor e contribui para o avanço da fronteira do saber. 


\section{NOTAS}

1. Alguns autores se referem como Indústria de Defesa e Segurança, haja vista que defesa é vocacionada para defesa externa e segurança para defesa interna e, obviamente, possuem áreas idênticas ou similares. No caso desse artigo, somente será tratado o problema da defesa.

2. Assim chamadas as grandes empresas que, por intermédio de parcerias e fornecedores, se responsabilizam diante do cliente a atender suas demandas. 3. Programa Nacional de Desenvolvimento de Submarinos, responsável pelos projetos de construção de 4 submarinos convencionais e um de propulsão nuclear brasileiros.

\section{Engenharia Logística, segundo o Council of Logistics Engineering} Professionals (CLEP), dos EUA, é "o campo da engenharia que visa a organização científica das compras, transportes, armazenamento, distribuição e armazenagem de produtos acabados."

5. Instituto Internacional de Estudos para a Paz.

6. A BID reúne o conjunto das empresas estatais e privadas, bem como organizações civis e militares, que participam de uma ou mais das etapas de pesquisa, desenvolvimento, produção, distribuição e manutenção de produtos estratégicos de defesa. Um novo incentivo à BID foi introduzido através da Lei 12.598/2012, marco legal para as compras, as contratações e o desenvolvimento de produtos e sistemas de defesa no país. A iniciativa está inserida no contexto do plano Brasil Maior. (Brasil, 2012). A Lei 12.598/2012 assinala um ponto de inflexão no modo como o Brasil cuida da indústria de defesa.

7. Conhecido como off set.

8. Tais como: salinidade, umidade, alta pressão, desgastes mecânicos e eletrônicos, eventuais avarias, necessidade de atualização de HW e SW e variação de temperatura constante, dentre outras.

9. Departament of Defense (DoD). Atualmente, o sistema de aquisição desse órgão é a base para a maioria das Forças Armadas do mundo, haja vista ter sido testado e validado desde o período da Guerra Fria até os conflitos no Iraque e Afeganistão.

10. O Departamento de Defesa dos EUA utiliza a padronização como benefício para a aquisição de interoperabilidade, custos, compatibilidade logística entre sistemas e outros aspectos.

11. Itálico nosso.

12. Documento enviado para fornecedores potenciais para fornecimento 
de informações que serão utilizadas no processo de pré-qualificação ou na elaboração de uma RFP. É mais genérico que uma RFP e não trata de questões comerciais, visa a aprimorar os conceitos técnicos de uma determinada necessidade do cliente.

13. Conjunto de documentos enviado aos fornecedores potenciais para elaboração de propostas técnicas e comerciais. Descreve detalhadamente sistemas ou especificações de produtos, solicitando, inclusive, aspectos de ALI para a operação assistida e manutenção.

14. Nível de serviço.

15. Associação Francesa de Normatização.

16. Expressão em inglês utilizada na logística, cujo significado é a entrega de um bem ou serviço no ponto final de entrega. Muitas vezes, essa fase do transporte é a mais complexa e problemática, como distribuição urbana, em portos e áreas isoladas.

17. CAPEX é a sigla da expressão inglesa capital expenditure, e significa despesas de capital ou investimento em bens de capital. É o valor a ser pago na aquisição de um determinado sistema de defesa e tecnologia.

18. OPEX é a sigla da expressão inglesa operational expenditure, e significa custo ligado à manutenção dos equipamentos que compõem o sistema e aos gastos de consumíveis e outras despesas necessárias ao funcionamento e à manutenção sistema de defesa e tecnologia.

19. Commercial off-the-shelf. Termo em inglês que significa sistemas, subsistemas e componentes que podem ser facilmente adquiridos no mercado.

20. Na maior parte das vezes, extremamente caros e que não podem ser devolvidos caso não sejam utilizados, por não serem COTS.

21. Significa Reability Centered Maintenance, ou, em português, Manutenção Centrada na Confiabilidade. Desenvolvido, inicialmente, para a indústria aeronáutica, é utilizado, atualmente, na área de defesa e tecnologia.

22. Em português, significa Análise dos Modos de Falha, Efeitos e Criticalidade. Tem sua origem na indústria automobilística, na década de 1970. Também conhecida como Military Standard MIL-STD-1629A.

23. Umidade, refrigeração, controle de bactérias, planos de contingência para elementos radioativos e tantos outros quanto forem necessários.

24. A forma como se refere à aposentadoria dos militares.

25. Fica mais barato do que certificar depois.

26. Compra de passagens, hospedagem, alimentação e riscos de acidentes. 


\section{APOIO LOGÍSTICO INTEGRADO: PECULIARIDADES DA INDÚSTRIA DE DEFESA E TECNOLOGIA}

\section{RESUMO}

A indústria de Defesa e Tecnologia é caracterizada por inúmeras particularidades quando comparada ao mercado corporativo, dentre elas, a necessidade de um Apoio Logístico Integrado (ALI) robusto que vai além da entrega e envolve o ciclo de vida do sistema. O objetivo desse artigo é ressaltar os principais pontos do Apoio Logístico Integrado na área de Defesa, abordando suas peculiaridades e destacar o papel das empresas integradoras.

Palavras-chave: Logística. Apoio Logístico Integrado. Indústria de Defesa e Tecnologia. Sistemas de Defesa complexos.

\section{ABSTRACT}

Defense and Technology Industry is characterized by numerous peculiarities compared to the corporate market among them the need for a robust Integrated Logistics Support (ILS) that goes beyond the delivery and involving life cycle of the system. The purpose of this article is to highlight the main points of the Integrated Logistics Support in the area of Defense and Technology, addressing their peculiarities and highlight the role of main contractors.

Keywords: Logistics. Integrated Logistics Support (ILS). Defense and Technology Industry. Complex Defense Systems.

\section{REFERÊNCIAS}

A Blanchard, B. S. (2008) System Engineering Management. Blacksburg, Virginia: Jonh Wiley \& Sons, Inc.

Brasil. (2013) "Declaração de Intenções entre o Ministério da Defesa da República Federativa do Brasil e o Serviço Federal de Cooperação TécnicoMilitar da Federação Russa Relativa à Cooperação em Defesa Antiaérea”. Atos assinados por ocasião da VI Reunião da Comissão de Alto-Nível de Cooperação BrasilRússia, 19 a 21 de fevereiro de 2013. Disponível em: http://www2.planalto.gov. $\mathrm{br} /$ vice-presidencia/vice-presidente/noticias/noticias-do-vice/2013/02/2012- 
RBED, v. 2, no 1 , jan./jun. 2015

02-20-michel-temer-russia-atos-assinados.

Brasil. (2012) "Lei no 12.598, de 21 de março de 2012. Estabelece normas especiais para as compras, as contratações e o desenvolvimento de produtos e de sistemas de defesa; dispõe sobre regras de incentivo à área estratégica de defesa; altera a Lei no 12.249, de 11 de junho de 2010; e dá outras providências. Conversão da Medida Provisória n ${ }^{\circ}$ 544, de 2011.” Presidência da República, Casa Civil, Subchefia para Assuntos Jurídicos. Disponível em: http://www.planalto.gov. br/ccivil_03/_ato2011-2014/2012/Lei/L12598.htm.

Brasil. (2003) EMA-400 - Manual de Logística da Marinha. Estado-Maior da Armada, 2a Rev., Brasília.

Correa Filho, Sérgio Leite Schimitt et al. (2013) "Panorama sobre a indústria de defesa e segurança no Brasil”. BNDES Setorial, Defesa, 38, p. 373-408. Disponível em: http://www.bndes.gov.br/SiteBNDES/export/sites/default/ bndes_pt/Galerias/Arquivos/conhecimento/bnset/set3810.pdf.

Diário do Pré-Sal (blogue) (2010). "Brasil planeja frota com seis submarinos nucleares e vinte convencionais”. Diário do Pré-Sal, 21 de novembro de 2010. Disponível em: https://diariodopresal.wordpress.com/2010/11/21/brasilplaneja-frota-com-seis-submarinos-nucleares-e-vinte-convencionais/.

Fleurant, Aude; Perlo-Freeman, Sam. (2014) "The SIPRI Top 100 ArmsProducing and Military Services Companies, 2013". SIPRI Fact Sheet, December 2014. Disponível em: http://www.sipri.org/research/armaments/production/ recent-trends-in-arms-industry/Fact\%20Sheet\%20Top 100\%202013.pdf.

Godoy, Roberto. (2014) "Caça mais caro estreia em bombardeio". O Estado de São Paulo, Internacional. 24 Setembro 2014. Disponível em: http://internacional. estadao.com.br/noticias/geral,caca-mais-caro-estreia-em-bombardeioimp-, 1565140 .

Jones, J. V. (2006) Integrated Logistics Support Handbook. Irvine, California: McGraw-Hill.

Sarti, F.; Ferreira, M. J. (2011) Agência Brasileira de Desenvolvimento Industrial. Diagnóstico: Base Industrial de Defesa Brasileira. Campinas: NEIT-IE-UNICAMP. 